\title{
RECORDED BIRTH WEIGHTS OF UK PRETERM BABIES ARE FREQUENTLY INACCURATE
}

R. Alanoor, J. Jones, P. Clarke

Neonatal Unit, Norfolk \& Norwich University Hospitals NHS Trust, Norwich, UK

Aims: Measurement of accurate birth weight is a keystone for fluid management, drug dosing, growth assessment, and classification of preterm babies. Weights measured on admission frequently include various items of attached equipment. Our aim was to determine how birth weights are recorded for preterm babies admitted to UK neonatal units for intensive care.

Methods: Telephone survey of all UK neonatal units in October-December 2010. We asked the lead/ senior nurses how babies' birth weights are recorded in their units, and whether allowance was made for any additional equipment attached.

Results: We obtained data from $208(100 \%)$ neonatal units contacted. Admission weights are recorded using bedside electronic scales in $144(69 \%)$ units, using in-incubator scales in $47(22 \%)$ units, and by either method in 14 (6\%) units. Remarkably, 3 (1\%) units (including one level 2 unit) said that for preterm babies who arrive already intubated from the delivery unit, an estimated birth weight is used. Only 54/208 (25\%) units take into consideration equipment attached to babies on weighing: 37/208(17\%) units have charts available to facilitate accurate deductions from babies' weights measured with equipment attached; 17 units routinely weigh separately all items of attached equipment and deduct this figure to obtain a baby's exact birth weight. 154/208 (74\%) units do not routinely take into consideration all attached equipment when assigning birth weights.

Conclusion: Birth weights assigned by the majority of UK neonatal units are overestimated. These data support the development of a standardised methodology for weighing premature babies at birth requiring intensive care. 